\title{
Feeling of doing in obsessive-compulsive checking
}

\author{
S. Belayachi ${ }^{\mathrm{a}, *}$, M. Van der Linden ${ }^{\mathrm{a}, \mathrm{b}}$ \\ ${ }^{a}$ Cognitive Psychopathology Unit, University of Liège, Belgium \\ ${ }^{\mathrm{b}}$ Cognitive Psychopathology and Neuropsychology Unit, University of Geneva, Switzerland
}

\section{A R T I C L E I N F O}

\section{Article history:}

Received 22 June 2009

Available online $\mathrm{xxxx}$

\section{Keywords:}

Obsessive-Compulsive Disorder

Checking

Sense of agency

Feeling of doing

Action-monitoring

\begin{abstract}
A B S T R A C T
Research on self-agency emphasizes the importance of a comparing mechanism, which scans for a match between anticipated and actual outcomes, in the subjective experience of doing.

This study explored the "feeling of doing" in individuals with checking symptoms by examining the mechanism involved in the experienced agency for outcomes that matched expectations. This mechanism was explored using a task in which the subliminal priming of potential action-effects (emulating outcome anticipation) generally enhances people's feeling of causing these effects when they occur, due to the unconscious perception of a match between primed and observed outcomes. The main result revealed a negative relationship between checking and self-agency for observed outcomes that were primed prior to actions. This suggests that checking individuals fail to grasp the correspondence between actual outcomes of their actions and expected ones. We discuss the possible role of undermined self-agency in checking phenomena and its relationship with cognitive dysfunction.
\end{abstract}

(c) 2010 Elsevier Inc. All rights reserved.

\section{Introduction}

Obsessive-Compulsive Disorder (OCD) is characterized by both obsessions (i.e., recurrent thoughts or images, particularly ones that cause distress) and compulsions (i.e., urges to perform mental or physical acts repeatedly). A fundamental characteristic of the OCD phenomenology is the peculiar way in which individuals with those symptoms construe what they are doing in certain behavioral situations. Current cognitive models suggest that compulsive behaviors are likely to be accompanied either by the aim of preventing threatening events (i.e., harm avoidance; Rachman, 1997; Salkovskis, 1985) or by a particular sensation "that something's wrong" with an action or the environment (incompleteness; Coles, Frost, Heimberg, \& Rhéaume, 2003). Interestingly, the prevalence of these two core dimensions seems to vary across the different subtypes of OCD: harm avoidance may particularly characterize washing and obsessing symptoms (Tolin, Brady, \& Hannan, 2008), while incompleteness may be especially associated with checking (Coles et al., 2003; Tolin et al., 2008) and ordering (Ecker \& Gönner, 2008).

Incompleteness and "not quite right" feelings are characterized by impressions of failure or imperfection, which can lead to an inability to achieve a sense of "task completion" or "closure" regarding actions (e.g., locking the door) or perceptions (e.g., objects on a table). In the particular case of action incompleteness (i.e., checking behaviors), people may experience inconsistent feelings that "actions or intentions have been incompletely achieved" (Summerfeldt, Huta, \& Swinson, 1998, p. 80), or feel only a weak sense of goal satisfaction. Such conflicting appraisals of one's behaviors have been related to impairments of the ability to monitor actions (Fitzgerald et al., 2005; Gehring, Himle, \& Nisenson, 2000; Maltby, Tolin,

\footnotetext{
* Corresponding author. Address: Cognitive Psychopathology Unit, Department of Cognitive Sciences, University of Liège, Belgium. Fax: +32 43662808. E-mail address: sanaa.belayachi@ulg.ac.be (S. Belayachi).
} 
Worhunsky, O’Keefe, \& Kiehl, 2005; Pitman, 1987; Ursu, Stenger, Shear, Jones, \& Carter, 2003). For example, Pitman (1987) proposed that compulsive behaviors may stem from a recurrent perception of a "mismatch signal" informing one of a discrepancy between actual outcomes and intended effects; checking symptoms have been consistently connected with abnormal action-monitoring (Hajcak \& Simons, 2002).

The role of action-monitoring is to assess the extent to which ongoing actions and subsequent outcomes match up to what was initially intended (i.e., internal goals and plans), and to trigger either a matching signal (i.e., goal satisfaction) or a mismatch signal, when goals may not be attained or expected effects may not be obtained (Aarts, Custers, \& Wegner, 2005; Pitman, 1987; Ridderinkhof, van den Wildenberg, Segalowitz, \& Carter, 2004). In addition, by triggering those informing signals, action-monitoring plays an important role in the subjective understanding of "what one is doing" or "what one has just done"; a dysfunction at this level may thus lead to an inconsistent appraisal of one's action. More concretely, those matching signals may generate an internal feeling of doing; this "impression" would in turn constitute the phenomenal cue of goal attainment, on the basis of which people are able to naturally end their action (Aarts et al., 2005; Woody et al., 2005). Hence, an inability to generate a matching signal when observed effects correspond to expected ones may undermine the experience of having actually performed those intended effects. From this perspective, lacking such a basic "feeling of doing" could make people less sure of whether they have actually satisfied a goal and cause them to feel incompleteness, which may in turn trigger adjustment behaviors to ensure that the goal has been completed (i.e., checking behaviors). The present study therefore aimed to explore checking individuals' ability to generate a "feeling of doing" for outcomes that obviously match expected effects.

Theories of the sense of agency (i.e., the subjective experience of doing) offer a reliable theoretical context for understanding how such a basic feeling of doing can be modulated by the unconscious perception of a correspondence between observed action-effects and expected ones (Aarts, Wegner, \& Dijksterhuis, 2006; Aarts et al., 2005; Wegner \& Wheatley, 1999). Experience of agency may arise when one feels that a perceived outcome (e.g., a locked door) is caused by one's action (e.g., putting a key in the lock), and that both actions and their related effects correspond to mental representations (e.g., the desire to secure the house), which are generally intentions, goals and expected outcomes (Aarts et al., 2005, 2006; Wegner, 2002; Wegner \& Sparrow, 2004; Wegner \& Wheatley, 1999).The perception of a match between perceived outcomes and anticipated ones would trigger the basic feeling that we had caused some intended effects through our actions. Accordingly, people may feel that they have caused specific effects whenever these effects are mentally anticipated (Wegner \& Sparrow, 2004); so long as an unconscious mechanism is capable to grasp a correspondence between anticipated and observed effects and subsequently to trigger a matching signal (Aarts et al., 2005, 2006).

In fact, research on the sense of agency has demonstrated that the experience of agency can be artificially induced in tasks in which participants' behavioral outcomes coincide with prior consistent thoughts about those outcomes. For example, Aarts et al. (2005) demonstrated that priming action-effects just prior to participants' actions enhanced the feeling that they had caused those effects when they actually occurred. Their work was based on the assumption that subliminal priming mimics the automatic activation of the representations of action-effects before the action, while simultaneously preventing conscious awareness of these thoughts. In their study, the authors used a task in which the participant and the computer each moved a square in opposite directions; the participants' task was to press a key (i.e., move) to stop the motion of the squares (i.e., "actual outcome"), and subsequently to determine whether they or the computer could have caused the square to stop in the observed position (i.e., agency judgment). In reality, the participants did not have any control over causing the square to stop in that particular position, which was arranged so that it never represented either the participants' or the computer's real stop position. In half of the trials, the square position to be presented was primed just before the participants stopped the motion of the square (i.e., prior thoughts on expected effect), and the results showed that effect-priming significantly enhanced the "feeling of personally causing" the square to stop in the presented position. In this priming task, people's illusion of agency is thought to reflect the automatic comparison between observed and anticipated effects, leading to a matching signal that may be responsible for the increased impression of having caused the observed outcomes. Thus, this task allows the assessment of the ability to grasp a correspondence between observed and expected effects, on the basis of which one may subsequently experience a feeling of agency for those effects.

To sum up, the perception of a match between expected effects and observed effects of actions tends to make people more prone to experience a feeling of doing for those effects (Aarts et al., 2006; Wegner \& Sparrow, 2004). Furthermore, this mechanism is claimed to be responsible for the illusion of agency observed in experimental settings whenever perceived outcomes are primed just before the participants act (Aarts et al., 2005, 2006; Jones, de-Wit, Fernyhough, \& Meins, 2008). Consequently, an altered comparing process, leading to an inconsistent matching signal, should lead people to experience less of a "feeling of doing" in the agency judgment task developed by Aarts et al. (2005). Consistently, checking symptoms seem to be specifically related to abnormal action-monitoring, which may lead to inconsistent mismatch signals, in addition to feelings of incompleteness (e.g., Hajcak \& Simons, 2002). Furthermore, it has been proposed that individuals' feelings of having an unfulfilled goal, which characterize checking, may be related to the inability to generate an internal signal that normally triggers a basic sense of task completion (Szechtman \& Woody, 2004).

From this perspective, individuals with checking symptoms may have a comparing mechanism that is less efficient at grasping an accurate match between real outcomes and expected ones, which in turn leads checkers to lack any feeling of doing for those outcomes. The methodology of Aarts et al. (2005) allows one to examine this issue by means of the level of experienced agency for outcomes that have been primed prior to participants' action (i.e., in the priming of the outcome condition of the agency judgment task). Indeed, the illusion of agency in this condition has been interpreted as resulting from the perception of a match between primed and observed outcomes, and the generation of a matching signal by the 
comparing mechanism. Considering that a defective comparing mechanism would undermine experienced agency in the priming condition, and if checking symptoms are indeed connected with such an impairment, we could expect that those symptoms would be related to lower experienced agency in the priming condition of the agency task. Since "not just right" experiences in ordering symptoms may concern perception rather than action, and the other OCD dimensions seem not to be phenomenologically related to the action component explored in this study, we expected that checking symptoms appear specifically associated with lower experience of agency for observed outcomes that match "anticipated" ones (because of subliminal priming), as compared to the other OCD dimensions.

Those issues will be examined in individuals with subclinical levels of OCD symptoms, as non-clinical participants with checking proneness have been found to have clinical features and cognitive impairments similar to those identified in checking patients (Muris, Merckelbach, \& Clavan, 1997; Zermatten, Van der Linden, Larøi, et al., 2006).

\section{Method}

\subsection{Participants}

One hundred and twenty-eight undergraduates from the University of Liège, aged between 18 and 36, participated in the study. Participants were randomly recruited from various faculties and schools of the university (i.e., Law and Political Science, Psychology and Education, Science, Applied Science, Management, Social Sciences); they were not compensated for their participation. Data from three participants were omitted because they did not respect the instructions. In addition, the debriefing revealed that 16 participants may have realized the true nature of the study during the task (see the debriefing description in Section 2.4.); those participants' data were excluded from the analysis. Data from two participants also had to be discarded as they appeared to be critical outliers (cf. Section 3). The reported results are from the remaining 107 participants (39 males and 68 females). Their mean age was 22.5 years $(S D=3.14$ years).

\subsection{Experimental task and procedure}

Informed consent was obtained from all participants following a full explanation of the experimental procedure. Detailed written and oral instructions explained that we were interested in people's feelings of control and that participants would be asked to direct the movement of a black square on a screen. In an individual testing session, participants performed the illusion of agency task (i.e., the Wheel of Fortune Task) and completed two questionnaires assessing OCD and depression symptoms, as well as questionnaires unrelated to the present study. Half of the participants first undertook the Wheel of Fortune Task, and then completed all the questionnaires; the other half completed the questionnaires before the task. The order of the questionnaires was counterbalanced across participants. Participants were debriefed at the end of the testing session.

The illusion of agency task was programmed in the Matlab environment; we used the same task administration and scoring described in Aarts et al. (2005). The task consisted of 16 trials in which participants first had to press down and hold the $S$ key of the keyboard to cause a gray square to move rapidly in a rectangular path in a counter-clockwise direction. At the same time, another gray square ("under the computer's control") moved along the same path but in a clockwise direction. At a random point in time, the gray squares could no longer be seen on the screen and participants had to press the Enter key to stop the motion of the squares they could no longer see but which were said to still be rotating. Immediately after the Enter key press, a black square appeared in a specific location on the path (i.e., representing the potential outcome of the participants' action of pressing the Enter key), and participants had to indicate whether they had caused the square to stop in that position. In eight trials (priming of outcomes condition), the black square's position was primed just before the participants pressed the Enter key, while there was no priming of effects in the other eight trials (no priming of outcomes condition). The order of trials with and without priming of potential outcomes was randomly specified (for each participant, the computer randomly determined the order of the 16 trials at the beginning of the task). The different events in each trial were as follows:

\subsubsection{Setting the square in motion}

Each trial began with a 3-s warning signal (the word "Warning" in French appeared on the screen); then, the message "Start" appeared in the center of a rectangular path, made out of eight white squares, until the participant pressed the $S$ key. The participant's and the computer's squares then began to move along the path in alternate steps (the squares were presented one after the other). In fact, the white squares representing the eight different positions on the path turn gray whenever one of the squares passes through them; each gray square was displayed for $60 \mathrm{~ms}$ in each position. Thus, it took $960 \mathrm{~ms}$ for one complete lap to occur $(60 \mathrm{~ms} \times 8$ positions $\times 2$ [participant's and computer's] squares; Aarts et al., 2005). The number of laps that occurred in a trial was programmed to vary randomly between 8 and 10 . In each condition, the starting location of the squares varied among all possible locations throughout the eight trials, and each possible starting position was randomly selected.

\subsubsection{Priming event}

In the priming of outcomes condition, the black square that was to be displayed (i.e., potentially representing the participants' action-effect) was flashed on the screen before the appearance of the message "Stop." The subliminal priming of 
the black square position occurred $40 \mathrm{~ms}$ after the last presentation of the participant's square, for $34 \mathrm{~ms}$; $46 \mathrm{~ms}$ later, it was followed by the message "stop." The primed location was always the same as the subsequently presented position of the black square. In the no priming of outcomes condition, the position of the black square was not flashed (the position was presented in white the whole time). The priming event was employed for every possible location, resulting in eight replications of each condition.

\subsubsection{Stopping the square}

At a random point in time (between 8 and 10 laps of the path), the word "Stop" appeared in the center of the grid until the participant pressed the Enter key to stop the motion of the square. When the message "Stop" appeared on the screen, only the eight empty white tiles were shown: the gray squares could no longer be seen but participants were told that the squares were still continuing to move around the path and that they had to stop the progress of their square. The participants' action (pressing the Stop button) resulted in one of the white squares of the grid turning black, which was said to represent the position of either their own square, or the computer's, at the time they pressed Stop. This black square appeared $100 \mathrm{~ms}$ after the Enter key press, and was presented for $1 \mathrm{~s}$. The location of this square was always four positions farther than the last position of the participant's square before the Stop message had appeared, so that they had no actual control over where the black square landed. In each condition (the priming of outcomes and no priming of outcomes conditions), this stop location occurred in each of the eight tiles of the path.

\subsubsection{Agency ratings}

Immediately after the presentation of the black square, participants were asked to indicate whether they felt they had caused the square to stop on that position or whether the computer had. Agency ratings were made on a 10-point Likert scale that appeared on the screen, running from "not me at all" (0) to "absolutely me" (9). Participants were told that a "not me" response was represented by values from 0 (if they were certain) to 4 (if they were not sure), while points from 5 to 9 represented a "me" response, with 5 if they were not sure, and 9 if they were certain. They were also asked to use intermediate values to moderate their judgment and to rely on their feelings if they had difficulties generating a response.

\subsection{Measures}

\subsubsection{Experienced agency}

For reasons of ease, participants were asked to rate their feelings of causing the square to stop in that position by using the 0-9 keys of the number pad; these agency ratings were subsequently rescaled on a 1-10 scale, for each of the 16 trials. Mean ratings of experienced agency were calculated for both the priming of outcomes condition and for the no priming of outcomes condition.

\subsubsection{Priming sensitivity index}

As agency ratings in the priming and no-priming conditions provide information only about the extent to which participants self-attributed the origin of outcomes; we also used the difference in agency ratings between the two conditions to quantify the amount of the increase in experienced agency due to the prior priming of potential outcomes (i.e., the effect of priming of outcome). Thus, for each participant, we computed a priming sensitivity index, defined as the mean agency ratings in primed trials less mean agency ratings in unprimed trials. Mean scores on the priming sensitivity index may range from -9 to 9 .

\subsubsection{Potential control}

We used the participants' reaction times (in ms) between the Stop message and the Enter key press (i.e., stopping the square) as a measure of the probability that they had actually stopped their "invisible but still rotating square" in the same position as that indicated by the black square (i.e., potential control "over producing the outcomes"). As Aarts et al. (2005) mentioned, it would take $330 \mathrm{~ms}$ after the presentation of the Stop message to land on the position of the black square; thus, if the participants pressed the Enter key $330 \mathrm{~ms}$ after the presentation of the Stop message, they would have landed on the position of the black square (see Aarts et al., 2005, for further explanation of this measure). For each participant, we calculated a measure of potential control, defined as the absolute difference between the response time after the Stop message and the time required to land exactly on the position of the black square $(330 \mathrm{~ms})$. The smaller the difference, the more likely that they actually could have caused the square to land on that position (see Aarts et al., 2005).

Trials in which reaction times were greater than $960 \mathrm{~ms}$ (i.e., if the participants pressed the Enter key after the invisible rotating square had started to make a new lap since the last presentation of their gray square) were first corrected, by subtracting $960 \mathrm{~ms}$ (i.e., the time for a complete lap to occur) from those slower responses, then the difference between this new response time and $330 \mathrm{~ms}$ was calculated (Aarts et al., 2005). Trials in which participants' reaction times were greater than 1920 ms were removed from the data (Jones et al., 2008); they represented 3.9\% of the total trials in the whole sample.

\subsubsection{Obsessive-Compulsive Inventory - Revised (OCI-R, Foa et al., 2002)}

The validated French version of the OCI-R (Zermatten, Van der Linden, Jermann, et al., 2006) is a self-report questionnaire that consists of 18 items evaluating OCD symptoms and is composed of six subscales: "Washing," "Obsessing," "Ordering," 
"Checking," "Hoarding," and "Neutralizing." Participants were asked to determine to what extent the situation described in each particular statement had distressed them during the past month, using a 5-point scale ( $0=$ "not at all"; $4=$ "extremely"). Total scores range from 0 to 72; the OCI-R subscales scores both range from 0 to 12 . Cronbach's alphas indicated good to acceptable internal consistency for all the measures (OCI-R total score: .81; Checking: .77; Washing: .72; Obsessing: .71; Ordering: .84; Hoarding: .66; Neutralizing: .52). The range of scores obtained on each OCD measure included scores comparable to those observed in clinical samples (OCI-R total score: 1-46; Checking: 0-10; Washing: 0-12; Obsessing: 0-10; Ordering: 0-11; Hoarding: 0-11), except for the Neutralizing OCI-R subscale (0-7). Mean scores for OCD dimensions are shown in Table 1 and revealed that Ordering symptoms were the most frequent, while Washing dimensions were less frequently observed in a non-clinical sample, replicating previous studies of OCD symptoms in the general population (Zermatten \& Van der Linden, 2008a). However, statistical tests of homogeneity revealed that the mean Washing, Obsessing, Hoarding and OCI-R total scores are higher in our study than in a French-speaking sample of 220 undergraduates in Zermatten and Van der Linden's (2008a) ( $p s<.01$ ); whereas mean Neutralizing, Ordering and Checking scores were comparable across the two studies ( $p s>.25$ ). There was no gender difference for all those measures ( $p s>.08$ ).

\subsubsection{Center for Epidemiological Studies Depression Scale (CES-D, Radloff, 1977)}

The French adaptation of the CES-D (Fuhrer \& Rouillon, 1989) was used to assess participants' proneness to depression. This self-report measure consists of 20 items describing states of happiness or depressed mood. For each item, participants had to rate the extent to which this situation applied to them during the last week, by using a 4-point Likert scale, ranging from $0=$ "Never, rarely (less than 1 day)" to $3=$ "Frequently, all the time (from 5 to 7 days)." Responses to items that described states of well-being were reversed; total scores range from 0 to 60 . The French version of the CES-D has good overall psychometric properties and a factorial structure similar to that observed in the original English version (Fuhrer \& Rouillon, 1989). As indicated by Cronbach's alpha (.89), this scale has a good internal consistency. A statistical test of homogeneity confirmed that the mean score on the CES-D obtained in this study (see Table 1) did not differ from that observed in a French-speaking sample of 291 undergraduates in Riddle, Blais, and Hess's (2002) study ( $p>.52$ ). There was no gender difference $(p>.13)$.

\subsection{Debriefing}

Following the procedure in the Aarts et al. (2005) study, participants were debriefed after the completion of the entire protocol. The debriefing began by asking the participants whether they had "seen something special during the task," in order to ensure that primed locations were not perceived. We noted any response that referred to the perception of a flash or another square before the stop message; those participants were treated as having perceived the prime. Participants' answers were followed by the information that the presented black square sometimes appeared very quickly on the screen just before the stop message. This information was followed by a question about whether they had seen it or not, depending on the participants' reaction (e.g., an assenting reaction was followed by the question: "you did notice it?", while the question "you did not notice it?" was asked if participants had no reaction or were surprised). Participants who did not explicitly report having seen the black square flash in response to the first question but admitted in response to the second that they had seen it at least once were treated as having perceived the prime. The debriefing indicated that six participants had realized the true nature of the study because the paradigm was presented in social psychology courses they had taken. In addition, 10 participants reported having seen a black square flash (subliminal priming) in at least one trial and a few of them noted that the position of this square was identical to the location of the final square. Those participants' data were excluded from the analysis (see above), as their experience of the task was clearly not comparable to that of the other participants.

Table 1

Mean scores and SDs for the experienced agency (priming and no priming of outcome conditions), priming sensitivity, OCD, and depression measures for the whole sample and for each checking group.

\begin{tabular}{lcc}
\hline Dependent variables & Whole sample score $(S D)$ & Non-checking group \\
\hline Agency task & & \\
Priming of outcome condition & $5.1(1.1)$ & $5.6(1.12)$ \\
No priming of outcome condition & $4.6(1.2)$ & $4.8(1.42)$ \\
Priming sensitivity index & $1.1(0.9)$ & $0.88(1.32)$ \\
OCD dimensions & & $4.6(0.83)$ \\
OCI-R checking & $2.2(2.1)$ & $0.96)$ \\
OCI-R washing & $1.7(2.2)$ & $1.1(2.26)$ \\
OCI-R obsessing & $3.0(2.5)$ & $1.9(2.25)$ \\
OCI-R hoarding & $4.0(2.6)$ & $2.4(1.65)$ \\
OCI-R ordering & $3.8(2.9)$ & $2.2(2.12)$ \\
OCI-R neutralizing & $1.0(1.5)$ & $0.4(1.34)$ \\
OCI-R total score & $15.7(8.5)$ & $8.3(1.70)$ \\
Depression & $15.8(9.3)$ & $3.8(2.71)$ \\
\end{tabular}

Note: $S D$ : standard deviation. 


\section{Results}

The six OCI-R subscales were not normally distributed; those data were transformed using the Box-Cox transformation to comply with normality and homogeneity of variance. Multivariate outliers were checked using the Cook's distance index. Fox (1991) proposed to define multivariate outliers as cases that have a Cook's distance greater than the cutoff $4 /(n-k$ - 1) (where $n$ is the number of cases and $k$ is the number of predictors). Preliminary analysis identified two participants as multivariate outliers, with a Cook's distance $>0.04$ (i.e., 4/[109-8-1]; we decided to exclude data from these two participants.

\subsection{Preliminary analysis}

\subsubsection{Effect of outcome priming on experienced agency in the whole sample}

Mean ratings of experienced agency were calculated for both the priming of outcomes condition and the no priming of outcomes condition. The sample's mean scores for those measures are shown in Table 1. Statistical homogeneity tests confirmed that the mean agency judgments on primed trials and on non-primed trials obtained in this study did not differ from those observed in a sample of 143 undergraduates in the Jones et al. (2008) study ( $p s>.20$ ); they were also similar to that observed in the Aarts et al. (2005) sample of 50 participants ( $p s>.06$ ).

Because Jones et al. (2008) had found a gender effect on the illusion of agency - priming of outcomes enhanced feelings of agency in women but not in men - we performed a 2 (Outcome: Primed vs. Unprimed) within-participants $\times 2$ (Gender: male vs. female) between-participants ANOVA. Effect size was tested through the $f$ estimate, as suggested by Cohen (1988); according to Cohen's rule of thumb, an effect size of .10 is a small effect, .25 a medium effect and .40 a large effect. For each effect, the proportion of variance explained was left in parentheses $\left(\eta^{2}\right)$.

This analysis revealed a significant main effect of priming, suggesting that participants' agency feelings for observed outcomes were higher when they were primed just before their actions than when they were not primed at all, $F(1,105)=9.79$, $p=.002, f=.31\left(\eta^{2}=.09\right)$. This effect size is medium to large by Cohen's (1988) criteria and is comparable to the effect sizes for effect-priming of $\eta^{2}=.09$ found by Aarts et al. (2006) and $\eta^{2}=.05$ reported by Jones et al. (2008). As in the Jones et al. (2008) study, there was no main effect of gender, $F(1,105)=2.03, p=.157$, while the interaction between priming and gender was found to be significant, $F(1,105)=6.45, p=.013, f=.25\left(\eta^{2}=.06\right)$. By Cohen's (1988) criteria, this is a small to medium effect, and is less comparable to the effect size for effect-priming of $\eta^{2}=.11$ found by Jones et al. (2008). Paired $t$-tests performed separately for each gender on agency ratings across the two conditions revealed that there was no effect of priming for men, $t(38)=0.33, p=.742$ ); for women, agency ratings were significantly higher for the priming of outcome than the no priming of outcome trials, $t(67)=5.05, p<.001$, Cohen's $d=.70$. This effect size is comparable, even higher, to that observed in the Jones et al. study (Cohen's $d=.59$ ). As well, $t$-tests performed for agency ratings on the primed and non-primed trials and the priming sensitivity index across genders showed that there were no gender differences on primed trials, $t(105)=-0.34, p=.732$, whereas men scored significantly higher than women on non-primed trials, $t(105)=2.58$, $p=.011$, Cohen's $d=.50$; this result is comparable to that observed in the Jones et al. study (Cohen's $d=.52$ ). In addition, women scored significantly higher than men on the priming sensitivity index $t(105)=2.54, p=.013$, Cohen's $d=.49$.

\subsubsection{Potential control over producing the effect}

For each trial, we calculated the absolute difference between the response time after the message to stop and the initial time required to land exactly on the position of the black square. The mean absolute difference scores for the outcomeprimed condition and the unprimed condition were then calculated and entered in a 2 (Outcome: primed, unprimed) $\times 2$ (Gender: male, female) mixed-design ANOVA. This analysis was conducted on Log-transformed means, because equal variances were not assumed for both the outcome-primed condition and the unprimed condition (based on Levene's tests of homogeneity of variance). This analysis revealed no effect of priming, $F(1,105)=2.34, p=.129$, indicating that priming of outcome did not affect participants' potential control over producing the effect; the mean absolute difference score was $70 \mathrm{~ms}(S D=81)$. There was a main effect of gender, $F(1,105)=4.55, p=.035, f=.20\left(\eta^{2}=.04\right)$, suggesting that men were more likely to stop their square closer to the final position of the black square on both kinds of trials. Finally, there was no interaction between gender and priming, $F(1,105)=1.53, p=.218$.

\subsection{OCD symptoms and feeling of doing}

\subsubsection{Correlation analyses}

We first calculated Pearson correlations for the priming sensitivity index and agency ratings in the priming and in the non-priming conditions with the measures of OCD (i.e., the six transformed OCI-R subscales and OCI-R total scores) and depression (i.e., CESD). To correct for multiple comparisons, a Bonferroni correction of $(0.05 / 24)=0.002$ was used. Those analyses are summarized in Table 2 . Consistent with our hypothesis, checking symptoms were found to be negatively associated with agency ratings in the outcome-primed condition, but only at an uncorrected significance $(p=.011)$. However, contrary to our hypothesis, there was no significant relationship between the overall benefit of priming and checking 
Table 2

Pearson correlations between OCD dimensions and experienced agency (priming of outcome condition), priming sensitivity index and depression.

\begin{tabular}{lcc}
\hline & $\begin{array}{l}\text { Experienced agency } \\
\text { (priming of outcome condition) }\end{array}$ & $\begin{array}{l}\text { Experienced agency } \\
\text { (no priming of outcome condition) }\end{array}$ \\
\hline OCD dimensions & & \\
OCI-R checking & $-0.24^{*}$ & $-0.27^{* *}$ \\
OCI-R washing & 0.01 & -0.14 \\
OCI-R obsessing & -0.02 & 0.11 \\
OCI-R hoarding & 0.02 & 0.15 \\
OCI-R ordering & -0.10 & 0.09 \\
OCI-R neutralizing & -0.06 & $-0.23^{*}$ \\
OCI-R total score & -0.12 & -0.01 \\
Depression & -0.05 & 0.05 \\
\hline
\end{tabular}

Note: There was no correlation significant at $p<.002$ (Bonferroni correction).

Indicates uncorrected significance at $p<.05$.

*** Indicates uncorrected significance at $p<.01$.

symptoms. In addition, checking and neutralizing symptoms negatively correlated with agency ratings in the non-priming condition at an uncorrected significance $(p s>.005)$.

\subsubsection{Regression analyses}

Considering the potentially confounding influences of correlations between symptoms (OCD subtypes and depression), zero-order correlations alone cannot determine the extent to which checking is specifically related to lower experienced agency, as compared to the other OCD dimensions. Linear regression analyses were therefore conducted to examine whether checking symptoms predicted lower experienced agency, when the other OCD dimensions and depression are statistically controlled. Because the preliminary analysis showed an effect of gender on some agency measures, gender was also controlled in each regression analysis; we set women at -1 and men at +1 , so that a positive association corresponds to men. Thus, three multiple regression analyses were conducted to evaluate the specific associations between OCD dimensions and agency measures, when other OCD dimensions, depression and gender were partialled out. The dependent variables were the agency ratings in the priming condition, agency ratings in the non-priming condition and the overall benefit of priming; the six OCD dimensions, depression and gender were simultaneously entered as independent variables.

Visual examination of the normal probability plot of the residuals and a Kolmogorov-Smirnov test for normal distribution of the standardized residuals suggested that there was no problem of heteroscedasticity for any of the regression analyses. Multicollinearity problems were checked by means of tolerance values (ranging from .66 to .88) and VIF values (ranging from 1.13 to 1.50 ). Those values suggest that there was no sign of multicollinearity, since VIF values over 2.5 and tolerance below .40 are considered as problematic (e.g., Allison, 1999).

All the regression analyses are summarized in Table 3; a Bonferroni-corrected level of .017 (.05/3) was used. Effect size was tested through the $f^{2}$ estimate, as suggested by Cohen (1988); according to Cohen's rule of thumb, an effect size of .02 is a small effect, .15 a medium effect and .35 a large effect. The results indicate that lower experienced agency in the outcomeprimed condition was predicted only by checking symptoms $\left(t=-2.49, p=.014, \beta=-.29, f^{2}=0.06\right)$, which according to Cohen's criteria represents small to medium effect. The overall benefit of priming was marginally predicted by gender $\left(t=-2.24, p=.027, \beta=-.22, f^{2}=0.05\right)$ and by ordering symptoms $\left(t=-2.09, p=.039, \beta=-.22, f^{2}=0.04\right)$, both in the reverse direction. According to Cohen, these two effects are small to medium. Finally, agency ratings in the non-priming condition was predicted by gender $\left(t=2.53, p=.013, \beta=.23, f^{2}=0.07\right)$, and by ordering $\left(t=2.33, p=.022, \beta=.23, f^{2}=0.06\right)$ and hoarding symptoms $\left(t=2.18, p=.031, \beta=.20, f^{2}=0.05\right)$, at an uncorrected significance. All these three observed effects are small to medium. In addition, checking symptoms also predicted agency ratings in the non-priming condition $(t=-3.33, p=.001$, $\beta=-.35, f^{2}=0.12$ ), but in the reverse direction and this effect size is small to medium.

\subsubsection{Group comparison}

To address more directly the question of whether checking individuals, as compared to non-checking participants, are characterized by a lower illusion of agency (i.e., by a lesser priming effect or none at all), two groups were created based on participants' checking subscores on the OCI-R. The checking group consisted of individuals whose scores fell within the top quartile of the distribution (score $>3 ; N=22$; six males and 16 females), and the non-checking group consisted of individuals with scores in the lowest quartile of the distribution (score $=0 ; N=31$; nine males and 22 females). Statistical homogeneity tests $(p s>.30)$ suggest that the OCI-R checking scores of our checking group $(M=5.68 ; S D=1.70)$ were comparable to the OCI-R checking scores of a non-clinical subgroup of checkers $(5.60 ; S D=1.96$; Zermatten \& Van der Linden, $2008 \mathrm{a})$ and of a clinical population of OCD patients $(M=4.83$; $S D=3.86$; Foa et al., 2002). The mean scores of each group on the measures of agency, OCD and depression are shown in Table 1.

In order to examine the effect of priming of outcome on experienced agency according to the level of checking symptoms, we performed a 2 (Outcome: Primed vs. Unprimed) within-participants $\times 2$ (Group: Checking vs. Non-checking) betweenparticipants ANCOVA. The gender was used as a covariate, with women set at -1 and men at +1 . Effect size was tested 


\section{Table 3}

Standardized, unstandardized regression coefficients, $t, p$ values, partial correlation and effect size for gender, depression, and the six OCI-R subscales regressed on agency ratings in the priming condition, agency ratings in the no-priming condition and the priming sensitivity index.

\begin{tabular}{|c|c|c|c|c|c|c|c|}
\hline & $B$ & SE B & $\beta$ & $t$ & $p$ & Partial $r$ & $f^{2}$ \\
\hline \multicolumn{8}{|c|}{ Priming of outcome condition } \\
\hline Gender & -0.04 & 0.22 & -0.02 & -0.20 & 0.840 & -0.02 & 0.00 \\
\hline Depression & -0.01 & 0.03 & -0.03 & -0.21 & 0.831 & -0.02 & 0.00 \\
\hline OCI-R checking & -0.86 & 0.35 & -0.29 & -2.48 & 0.015 & -0.24 & 0.06 \\
\hline OCI-R washing & 0.23 & 0.45 & 0.06 & 0.52 & 0.605 & 0.05 & 0.00 \\
\hline OCI-R obsessing & 0.03 & 0.24 & 0.01 & 0.11 & 0.915 & 0.01 & 0.00 \\
\hline OCI-R hoarding & 0.15 & 0.16 & 0.10 & 0.96 & 0.337 & 0.10 & 0.01 \\
\hline OCI-R ordering & -0.03 & 0.16 & -0.02 & -0.19 & 0.847 & -0.02 & 0.00 \\
\hline OCI-R neutralizing & 0.20 & 0.80 & 0.03 & 0.25 & 0.806 & 0.02 & 0.00 \\
\hline \multicolumn{8}{|c|}{ No priming of outcome condition } \\
\hline Gender & 0.36 & 0.14 & 0.24 & 2.62 & 0.010 & 0.26 & 0.07 \\
\hline depression & 0.00 & 0.02 & 0.01 & 0.07 & 0.948 & 0.01 & 0.00 \\
\hline OCI-R checking & -0.75 & 0.22 & -0.35 & -3.38 & 0.001 & -0.32 & 0.12 \\
\hline OCI-R washing & -0.09 & 0.29 & -0.03 & -0.31 & 0.759 & -0.03 & 0.00 \\
\hline OCI-R obsessing & 0.22 & 0.16 & 0.15 & 1.39 & 0.167 & 0.14 & 0.02 \\
\hline OCI-R hoarding & 0.22 & 0.10 & 0.20 & 2.12 & 0.036 & 0.21 & 0.05 \\
\hline OCI-R ordering & 0.24 & 0.10 & 0.23 & 2.35 & 0.021 & 0.23 & 0.06 \\
\hline OCI-R neutralizing & -1.00 & 0.51 & -0.20 & -1.95 & 0.054 & -0.19 & 0.04 \\
\hline \multicolumn{8}{|c|}{ Priming sensitivity index } \\
\hline Gender & -0.31 & 0.14 & -0.22 & -2.24 & 0.027 & -0.22 & 0.05 \\
\hline Depression & 0.00 & 0.02 & -0.02 & -0.15 & 0.879 & -0.02 & 0.00 \\
\hline OCI-R checking & 0.14 & 0.22 & 0.07 & 0.62 & 0.535 & 0.06 & 0.00 \\
\hline OCI-R washing & 0.22 & 0.29 & 0.08 & 0.77 & 0.446 & 0.08 & 0.01 \\
\hline OCI-R obsessing & -0.19 & 0.16 & -0.14 & -1.20 & 0.234 & -0.12 & 0.01 \\
\hline OCI-R hoarding & -0.09 & 0.10 & -0.09 & -0.91 & 0.366 & -0.09 & 0.01 \\
\hline OCI-R ordering & -0.21 & 0.10 & -0.22 & -2.09 & 0.039 & -0.21 & 0.04 \\
\hline OCI-R neutralizing & 0.99 & 0.51 & 0.21 & 1.93 & 0.057 & 0.19 & 0.04 \\
\hline
\end{tabular}

through the $f$ estimate (adjusted for the addition of a covariate), as suggested by Cohen (1988); according to Cohen's rule of thumb, an effect size of .10 is a small effect, .25 a medium effect and .40 a large effect. For each effect, the proportion of variance explained was left in parentheses $\left(\eta_{p}^{2}\right)$.

This analysis revealed a significant main effect of priming, suggesting that participants' agency feelings for observed outcomes were higher when they were primed just before their actions than when they were not primed at all, $F(1,50)=4.38$, $p=.041, f=.29\left(\eta_{p}^{2}=.08\right)$. This effect size is medium to large by Cohen's (1988) criteria. There was also a main effect of group, suggesting that checking participants' reported lower agency ratings than participants with no checking symptoms $F(1,50)=7.42, p=.009, f=.39\left(\eta_{p}^{2}=.13\right)$; by Cohen's (1988) criteria, this is a medium to large effect. There was an interaction between priming and group, although it was not significant, $F(1,50)=3.44, p=.070, f=.25\left(\eta_{p}^{2}=.06\right)$; this is a small to medium effect (Cohen, 1988). One-way ANCOVAs conducted on agency ratings on the primed and non-primed trials across groups showed that there were no group differences on non-primed trials, $F(1,50)=1.40, p=.241$, whereas checking participants scored significantly lower than non-checking participants on primed trials, $F(1,50)=12.67, p<.001, f=.50$ $\left(\eta_{p}^{2}=.20\right)$; this is a large effect by Cohen's criteria. Finally, repeated-measures ANCOVAs were carried out separately for each group to examine agency ratings across the two conditions. These analyses revealed that Non-checking participants' agency ratings were significantly higher for the priming of outcome than for the no priming of outcome trials, $F(1,29)=5.83, p=.022, f=.42\left(\eta_{p}^{2}=.17\right)$, which is a large effect by Cohen's criteria; the effect of priming failed to reach significance for the checking participants, $F(1,20)=1.80, p=.19$.

\section{Discussion}

This study aimed to examine the "feeling of doing" in individuals with checking symptoms, by assessing their ability to experience agency for outcomes that matched expectations. Our results can be summarized as follows. First, and in line with previous findings (e.g., Aarts et al., 2005; Jones et al., 2008), priming the potential outcomes of participants' actions generally enhanced experienced agency for those outcomes when they actually occurred (in the whole sample). Furthermore, there was a gender effect: women, but not men, showed a significant effect of priming on agency ratings, consistent with previous results (Jones et al., 2008). As expected, checking symptoms were found to be negatively related to experienced agency in primed trials; this effect was found to be specific to checking as compared to the other OCD dimensions, and once comorbid depression and gender were controlled for. In addition, checking symptoms appeared to be negatively associated with lower 
experienced agency in the no-priming condition; while ordering and hoarding symptoms were found to be related to higher agency ratings in no-prime trials. Finally, there was no relationship between checking symptoms and the priming sensitivity index, although ordering symptoms tended to be negatively associated with the overall benefit of priming, but not when corrected for multiple comparisons (i.e., at an uncorrected significance).

When we compared participants with high levels of checking symptoms to those without checking symptoms, there was a significant main effect of checking symptoms on agency ratings, suggesting that checking participants had overall undermined agency ratings across the two conditions. Further analyses revealed no differences between the two groups in terms of participants' judgment of self-causation when the priming of outcomes was absent (i.e., no-priming condition); however, checking participants scored lower than non-checking participants in the priming condition. Also, there was a trend towards significance for the interaction between checking and the priming effect, suggesting that priming did not enhance experienced agency in high-checking participants.

The finding that individuals with checking symptoms were less prone to experience agency for consistent action-effects supports the assumption that checking phenomena are characterized by an undermined "feeling of doing." Considering that, in everyday behaviors, the feeling of doing may constitute an important cue for goal satisfaction and subsequent action closure (Aarts et al., 2005; Szechtman \& Woody, 2004; Woody \& Szechtman, 2000; Woody et al., 2005), an attenuation of this kind of phenomenal cue could explain why checking individuals frequently experience incompleteness, "not quite right" feelings and doubts about goal achievement. From this perspective, checking symptoms constitute behavioral adjustments in order to get more convincing (explicit) cues about actual goal completion or to experience a complete feeling of doing (i.e., "just right feelings"). Furthermore, Aarts et al. (2005) suggested that experienced agency elicited in the illusion of agency paradigm may depend on an unconscious mechanism of comparison that triggers a matching signal whenever a correspondence between observed and anticipated effects (i.e., effect-priming prior to action) is perceived. Along the same lines, Pitman (1987) proposed that recurrent inconsistent mismatch signals may underlie repeated behaviors in OCD. Thus, our results support the hypothesized involvement of a dysfunctional comparing mechanism and related matching signals in checking symptoms, as well as the idea of an attenuated subjective experience in connection with action-monitoring difficulties (Szechtman \& Woody, 2004; Woody et al., 2005).

Consistent with our hypothesis, altered action awareness may be specific to checking and not the other OCD dimensions. Indeed, as expected, the other OCD dimensions did not share any specific relationship with the measures of experienced agency; ordering symptoms were found to be negatively associated with the overall benefit of priming, but not when corrected for multiple comparisons (i.e., at an uncorrected significance). The associations found between lower experienced agency and checking and, to a lesser extent, ordering symptoms are quite consistent with the two-dimensional model of OCD whereby those symptoms may be predominantly associated with a peculiar experience of incompleteness. Nevertheless, one might expect that OCD symptoms frequently associated with the overestimation of personal influence in the occurrence of random outcomes (i.e., obsessing and washing dimensions) would be characterized by an increased experience of agency for uncontrollable outcome that match prior thoughts. One possible explanation is that the exaggerated personal responsibility that may characterize washing and obsessing symptoms may depend on the valence of the content of preceding thoughts and uncontrolled events (i.e., threatening events vs. neutral outcomes, as in the present study). It should be noted that participants with OCD symptoms (regardless of OCD subtype) have been reported to experience an increased illusory sense of control when desired outcomes match actual outcomes; this effect appeared more marked with aversive stimuli than with neutral stimuli (Reuven-Magril, Dar, \& Liberman, 2008). In their study, Reuven-Magril et al. used a preprogrammed sequence of neutral and aversive images, for which participants had to control and shorten their duration (i.e., desired outcome) by finding the right combination of key presses (i.e., action); their perceived level of control was assessed at various points during the task. Participants did not have any actual control over the duration of the stimuli, as the presentation time gradually increased (i.e., increased discrepancy between desired and actual outcomes) throughout the first half of the task and then gradually decreased for the remaining stimuli (i.e., increased matching between desired and actual outcome). Their results suggest that OCD symptoms may be characterized by more compulsive-like repetitive patterns of action (i.e., using the same key presses) and by an increased sense of control for aversive and, to a lesser extent, neutral stimuli. The apparent contradiction between the results of our study and the findings of Reuven-Magril et al. (2008) may be related to some major differences between the illusion of agency task and the illusion of control task. First, the two tasks may differ regarding the kind of sense of control they assess. Indeed, a recent article on the contribution of sense of control on sense of agency proposed to distinguish between the "sense that one has to exert control to generate and maintain an appropriate action program" and the "sense that one feels in control of an action" (Pacherie, 2008, p. 20). The first form of control depends on the importance of mental and behavioral attempts and the adjustments necessary to reduce the discrepancies between expectations and outcomes (i.e., "effortful control," such as would be the case in disrupted or unfamiliar actions); in such effortful situations, it is the conscious effort itself that may enhance the sense that one is engaged in and causing actions. On the other hand, feeling in control of an action only requires the accessibility of the result of the unconscious comparisons between predicted and actual states ("effortless control," such as would be the case for routine or automatic actions; Pacherie, 2008). In light of this model, we could argue that, in the illusion of control task used by Reuven-Magril et al., feelings of control and self-causation are predominantly elicited by the effortful control situation created by the design of the task. In our study, on the other hand, the illusion of agency task elicited a sense of effortless control and thus feelings of self-causation were predominantly based on automatic and unconscious processes, similar to those observed during nonconscious goal pursuit (Aarts et al., 2005, 2006). 
Furthermore, a recent cognitive model of OCD (Moulding \& Kyrios, 2006, 2007) suggests the involvement of two controlrelated features: the need for control (i.e., the general motivation to be able to exert control over events) and the sense of control (i.e., beliefs in one's ability to attain or avoid specific outcomes through one's actions). According to this model, the level of desired control generally leads to the deployment of appropriate behaviors in order to achieve desired outcomes; the level of perceived control in a specific context may vary accordingly. While, in most people, sense of control is generally positively related to the desire for control, a significant discrepancy between desired level of control and perceived control in certain situations (i.e., a high need for control but a low-level of perceived control) may lead OCD individuals to develop strategies that will give them a satisfying "feeling that things are under control." Thus, it is possible that the Reuven-Magril et al. (2008) paradigm may offer a context favoring such an artificially inflated sense of control, while the task used in our study may emphasize a process that is potentially implicated in the undermined sense of control, which has been particularly related to checking symptoms (Moulding \& Kyrios, 2007). In addition, the Reuven-Magril et al. study may highlight the phenomenon whereby compulsive-like behaviors lead OCD individuals to experience high levels of control, rather than the process underlying anxiety and distress, which in turn trigger OCD symptoms. In other words, the "feelings of undesired end-state to be realized" that characterize some OCD symptoms and subsequently trigger avoidance strategies remain to be explored; thus, further studies should be conducted to explore the relationship between obsessing and washing symptoms and the overestimation of personal influence in the occurrence of magical and/or threatening outcomes (Pronin, Wegner, McCarthy, \& Rodriguez, 2006).

Another possibility is that harm avoidance could be related to a more conceptual component of action awareness, namely the feeling that one's conscious thought is the cause of one's action (Pacherie, 2008; Synofzik, Vosgerau, \& Newen, 2008; Wegner \& Sparrow, 2004). This "conceptual sense of agency" would be based on conscious inferences leading one to draw (illogical) causal links between one's thoughts, identified as intentions, and external outcomes (i.e., a kind of post hoc ergo propter hoc fallacy). Exaggerated responsibility for threat events, which in fact are not the result of one's own actions but did coincide with one's consistent thoughts, would reflect an impaired top-down processing of agency (Synofzik et al., 2008), leading to a bias towards the self in the attribution of the agency of an event. By contrast, checking symptoms would be related to a "phenomenal sense of agency," implying the feeling that one's movement is causing some specific effects. This component may depend on an unconscious matching signal between actual outcomes of movement and anticipated effects, which may stem from bottom-up processes (Synofzik et al., 2008). However, these assumptions are merely speculative and laboratory experiments are needed to explore the extent to which the sense of agency constitutes a common factor in OCD dimensions, with the possibility that impairment of specific components of this sense could lead to different patterns of OCD symptoms.

Before concluding, we should emphasize some limitations of the present study. First, our interpretation of the nature of the relationship found between experienced agency and checking symptoms is limited, as we did not control for the potential confounding effect of other factors, such as dissociation, anxiety, and attentional focus. For example, dissociative states, which are frequently associated with checking (Belayachi \& Van der Linden, 2009; Rufer, Fricke, Held, Cremer, \& Hand, 2006), can reduce awareness of action by altering the integration of action representations (e.g., expected effects) and the "present self in action" (e.g., observed outcomes) (Ansfield \& Wegner, 1996). Furthermore, several studies have emphasized the importance of attention focused on representations related to goals, expected effects or intention in action awareness, as compared to attention focused on movements (Lau, Rogers, Haggard, \& Passingham, 2004). Consistently with this idea, checking individuals seem to parse their habitual actions mainly with regard to movement parameters, rather than goals and outcomes (Belayachi \& Van der Linden, 2009). However, anxiety, which focuses attention on details and promotes local perceptual information processing, could alter the shift of attention towards effect representation (Boyer \& Liénard, 2006; Derryberry \& Reed, 1998). Thus, as we did not control for the impact of anxiety, attention and dissociation in this study, we cannot rule out the possibility that those factors may account, at least in part, for the relationship found between checking symptoms and low sense of agency. Further studies should therefore explore the potential confounding effect of those variables.

Furthermore, our results prevent us from determining the nature of the relationship between lower experienced agency and compulsive checking. Indeed, what remains unclear is whether lower experience of agency leads people to check their actions in order to ensure that they have been completed, or whether repeated checking of actions may lead people to deploy alternative strategies for authorship ascription. It is possible that a lack of matching signal accessibility may prevent people from experiencing success in achieving expected outcomes, which may in turn lead them to check their actions. Conversely, individuals with checking symptoms may rely on different cues for authorship processing, such as movement parameters. One way of answering this question would be to explore the extent to which priming the concept of success may increase feelings of self-causation in checking individuals, and the extent to which inducing checking may engender an undermined experienced agency and less confidence in self-agency. Nevertheless, we assumed that an impaired feeling of doing may constitute only one of the numerous factors implicated in checking, such as anxiety, attentional focus, reality monitoring difficulties and poor memory for actions (Boyer \& Liénard, 2006; Zermatten \& Van der Linden, 2008b). As mentioned above, anxiety and attentional focus may have a potential confounding effect as those variables may interfere with the normal processing of action-monitoring. Furthermore, an undermined immediate sense of doing could be responsible for reality monitoring difficulties and memory failure, which in turn may trigger doubts about action performance and checking symptoms. In this vein, it would be important to clarify the relationships between low feeling of doing, difficulties with reality monitoring and action memory. 
Another potential limitation is that the study also lacks a control for the impact of beliefs about self-agency on agency ratings. Indeed, checking symptoms correlated with lower experienced agency in both primed and non-primed trials; group comparisons consistently revealed a main effect of checking proneness on overall agency ratings, suggesting that checking participants reported lower agency ratings than those with no checking symptoms. In light of those results, one could argue that checking individuals have lower agency ratings in both the primed and non-primed conditions because they have an overall lack of confidence in their self-agency. Thus, an alternative explanation of our results would be that the relationship observed between authorship ascription and checking symptoms could be mediated by control beliefs such as self-determination, which have recently been found to constitute an additional source of information for authorship processing (Aarts, Oikawa, \& Oikawa, 2010). Consistently with this idea, OCD symptoms, and particularly checking symptoms, may be related to an undermined perceived control (beliefs about one's ability to perform an action and the extent to which that action will lead to a desired outcome or avoid an undesirable one; Moulding \& Kyrios, 2007). Thus, further studies are needed to explore the extent to which beliefs of control and self-determination may account, at least in part, for the relationship found between checking symptoms and lower self-agency. Nonetheless, group comparisons also revealed that checking participants scored significantly lower than non-checking participants on primed trials, while there was no group difference on non-primed trials, supporting the assumption that checking participants were less prone to base their agency on expected outcomes. However, there are limits to how to interpret unexpected results concerning agency ratings in the no priming of outcome condition as there is no assumption about the mechanisms that underlie agency judgments in the absence of "representation of action-effect"(i.e., the observed outcome was not primed prior action). The action control mechanism underlying agency ratings in the Wheel of Fortune Task may vary across the two conditions (e.g., conceptual congruency between preview and action-effect in the priming condition vs. congruency between sensory predictions made from the movement and the actual sensory consequence in the no-priming condition). Therefore further examinations of cues that might allow participants to correctly misidentify the self as the cause of observed outcomes in the no-priming condition would help to clarify some of our results.

Another intriguing outcome is that ordering symptoms were negatively correlated with priming sensitivity, whereas checking symptoms were not, although group comparisons revealed an absence of any effect of priming for the checking participants, as compared to non-checking participants. However, it is worth noticing that the negative association found between ordering symptoms and the priming sensitivity index is difficult to interpret. Indeed, this agency measure, which has been defined as the mean agency ratings in primed trials less mean agency ratings on unprimed trials, may range from -9 to 9 . Thus, negative scores suggest a negative effect of priming (i.e., resulting in lower agency ratings on primed trials than on non-primed trials), positive scores suggest a benefit of priming (i.e., resulting in higher agency ratings on primed trials than on non-primed trials), while a null score suggests an absence of any priming effect. However, we cannot determine the extent to which a negative correlation with this measure reflects a negative effect of priming on agency ratings, an absence of priming effect or a lower priming effect (i.e., a benefit of priming, but to a lesser extent). Thus, the results obtained from the priming sensitivity index and the related interpretations must be considered cautiously.

In addition, the OCI-R alone overlooks the motivational aspects of checking symptoms, whereas some studies have yielded evidence that checking symptoms are motivationally heterogeneous (e.g., Ecker \& Gönner, 2008; Tolin, Woods, \& Abramowitz, 2003). Thus, what remains unclear is whether checking symptoms, as measured by the OCI-R checking subscale, reflect a tendency to re-enact actions because of a lack of an immediate feeling of satisfaction (incompleteness) or compulsive checking of safety actions in order to avoid potential threat (harm avoidance). Also, it is possible that incompleteness feelings may trigger doubts about whether an action has been achieved, which in turn can lead to harm avoidance features whenever one becomes aware of the danger, if such doubts concern safety actions, and consequently lead to checking behaviors (i.e., incompleteness and harm avoidance). Such heterogeneity within the checking measurement may possibly account for the weak association we observed between checking symptoms and experienced agency. Further studies should be conducted in order to compare various subgroups of checkers according to their predominant motivation, as well as OCD subtypes within the same core motivational dimension, on the illusion of agency task.

Finally, another important limitation of this study is the relative weakness of the effect of checking proneness on undermined self-agency. In this regard, we should emphasize that our study does not control for major factors that potentially interfere with the relationship between checking symptoms and self-agency as elicited by the Wheel of Fortune Task, such as the way participants generally construe their actions (i.e., level of agency). Indeed, this factor has been related to both checking symptoms (Belayachi \& Van der Linden, 2009) and self-agency based on the Wheel of Fortune Task (van der Weiden, Aarts, \& Ruys, 2010). Level of agency stems from the Action Identification theory (Vallacher \& Wegner, 1985, 1989), which states that any action can be identified within a cognitive hierarchy of meanings, in which the lower-levels represent instrumental features and movement parameters, while the higher-levels relate to the desired goal and expected outcomes. Vallacher and Wegner proposed that the particular level at which an action is identified may reflect the particular representation (movements parameters vs. outcome) on the basis of which the action is monitored. Although the way an action is identified depends on several action-related features (e.g., action complexity, degree of expertise, action disruption or error), people tend to adopt a predominant level of action identification for various behaviors (i.e., level of agency; Vallacher \& Wegner, 1989). Thus, there are people who have a tendency to identify their actions according to related purpose and implications (i.e., a high-level of agency) and others who tend to identify their actions mainly regarding procedural aspects and motor subcomponents (i.e., a low-level of agency). Recently, van der Weiden et al. examined the relationship between the level of agency and the feeling of doing by using the Behavior Identification Form, a questionnaire designed to assess 
the level at which participants generally construe their habitual actions (i.e., mainly based on movement parameters vs. expected outcomes), in addition to the Wheel of Fortune Task. Their results suggested that the more people tend to generally identify their action according to related outcomes and pursued goals (i.e., high-level of agency), the more they experience self-agency for outcomes that have been primed prior actions. However, outcome priming did not increase agency ratings among participants who had a low-level of agency. Interestingly, checking symptoms have been previously found to be related to a low-level of agency, as assessed by the Behavior Identification Form (Belayachi \& Van der Linden, 2009). Furthermore, recent data (Belayachi \& Van der Linden, 2010), suggest that checking participants with no dysfunctional beliefs are those who are also characterized by a low-level of agency. Taken as a whole, one could argue that the rather small effect sizes in our study could be related to the lack of control of both the level of action construal and the motivational core feature of checking symptoms. Further studies are needed in order to clarify this issue.

In sum, our results suggest that individuals with checking symptoms have an impaired ability to experience agency for outcomes that match expectations, while the other OCD dimensions are not related to impaired agency. These results also suggest that an impaired sense of agency in checking symptoms is likely to be connected with an alteration of basic action processing involved in the phenomenal sense of acting and causing effects congruent to "what was intended." Overall, they are in agreement with the idea that abnormal action-monitoring, observed in both clinical and non-clinical samples, may underlie checking features. Nevertheless, although studies of non-clinical samples can generally be extrapolated to clinical samples, further studies should be conducted to replicate our findings in individuals with more severe OCD symptoms (i.e., OCD patients). In addition, further experiments are needed to explore the extent to which some OCD symptoms may be specifically related to another component of sense of agency potentially involved in altered personal causation in the occurrence of uncontrollable events. Thus, it would be interesting to explore whether the two core motivational dimensions in OCD may be at least partly explained by the component of the sense of agency that is predominantly impaired.

\section{Acknowledgment}

This study was supported by a Grant from the French-speaking community of Belgium (Action Recherche Concertée, Convention 06/11-340).

\section{References}

Aarts, H., Custers, R., \& Wegner, D. M. (2005). On the inference of personal authorship: Enhancing experienced agency by priming effect information. Consciousness and Cognition, 14, 439-458.

Aarts, H., Oikawa, M., \& Oikawa, H. (2010). Cultural and universal routes to authorship ascription: Effects of outcome priming on experienced self-agency in the Netherlands and Japan. Journal of Cross-Cultural Psychology, 41, 87-98.

Aarts, H., Wegner, D. M., \& Dijksterhuis, A. (2006). On the feeling of doing: Dysphoria and the implicit modulation of authorship ascription. Behaviour Research and Therapy, 44, 1621-1627.

Allison, P. D. (1999). Logistic regression using the SAS system: Theory and application. Cary, NC: SAS Institute Inc.

Ansfield, M. E., \& Wegner, D. M. (1996). The feeling of doing. In P. M. Gollwitzer \& J. A. Bargh (Eds.), The psychology of action: Linking cognition and motivation to behavior (pp. 482-506). New York: Guilford Press.

Belayachi, S., Van der Linden, M. (2010). Level of agency in subclinical checking: A dysfunctional beliefs-based effect, in preparation.

Belayachi, S., \& Van der Linden, M. (2009). Level of agency in sub-clinical checking. Consciousness and Cognition, 18, 293-299.

Boyer, P., \& Liénard, P. (2006). Why ritualized behavior? Precaution systems and action parsing in developmental, pathological and cultural rituals. Behavioral and Brain Sciences, 29, 1-56.

Cohen, J. (1988). Statistical power analysis for the behavioral sciences (2nd ed.). Hillsdale, NJ: Lawrence Erlbaum.

Coles, M. E., Frost, R. O., Heimberg, R. G., \& Rhéaume, J. (2003). "Not just right experiences": Perfectionism, obsessive-compulsive features and general psychopathology. Behaviour Research and Therapy, 41, 681-700.

Derryberry, D., \& Reed, M. A. (1998). Anxiety and attentional focusing: Trait, state and hemispheric influences. Personality and Individual Differences, 25, $745-761$.

Ecker, W., \& Gönner, S. (2008). Incompleteness and harm avoidance in OCD symptom dimensions. Behaviour Research and Therapy, 46, 895-904.

Fitzgerald, K. D., Welsh, R. C., Gehring, W. J., Abelson, J. L., Himle, J. A., Liberzon, I., et al (2005). Error-related hyperactivity of the anterior cingulate cortex in obsessive-compulsive disorder. Biological Psychiatry, 57, 287-294.

Foa, E. B., Huppert, J. D., Leiberg, S., Langner, R., Kichic, R., Hajcak, G., et al (2002). The Obsessive-Compulsive Inventory: Development and validation of a short version. Psychological Assessment, 14, 485-496.

Fox, J. (1991). Regression diagnostics. Thousand Oaks, CA: Sage Publications.

Fuhrer, R., \& Rouillon, F. (1989). La version française de l'échelle CES-D (Center for Epidemiologic Studies - Depression Scale). Description et traduction de l'échelle d'auto-évaluation. Psychiatrie et Psychobiologie, 4, 163-166.

Gehring, W. J., Himle, J., \& Nisenson, L. G. (2000). Action monitoring dysfunction in obsessive-compulsive disorder. Psychological Science, 11, 1-6.

Hajcak, G., \& Simons, R. F. (2002). Error-related brain activity in obsessive-compulsive undergraduates. Psychiatry Research, 110, 63-72.

Jones, S. R., de-Wit, L., Fernyhough, C., \& Meins, E. (2008). A new spin on the Wheel of Fortune: Priming of action-authorship judgements and relation to psychosis-like experiences. Consciousness and Cognition, 17, 576-586.

Lau, H. C., Rogers, R. D., Haggard, P., \& Passingham, R. E. (2004). Attention to intention. Science, 303, 1208-1210.

Maltby, N., Tolin, D. F., Worhunsky, P., O'Keefe, T. M., \& Kiehl, K. A. (2005). Dysfunctional action monitoring hyperactivates frontalstriatal circuits in obsessive-compulsive disorder: an event-related fMRI study. Neuroimage, 24, 495-503.

Moulding, R., \& Kyrios, M. (2006). Anxiety disorders and control related beliefs: The exemplar of obsessive-compulsive disorder (OCD). Clinical Psychology Review, 26, 573-583.

Moulding, R., \& Kyrios, M. (2007). Desire for control, sense of control and obsessive-compulsive symptoms. Cognitive Therapy and Research, 31, 759-772.

Muris, P., Merckelbach, H., \& Clavan, M. (1997). Abnormal and normal compulsions. Behaviour Research and Therapy, 35, 249-252.

Pacherie, E. (2008). The phenomenology of action: A conceptual framework. Cognition, 107, 179-217.

Pitman, R. K. (1987). A cybernetic model of obsessive-compulsive pathology. Comprehensive Psychiatry, 28, 334-343.

Pronin, E., Wegner, D. M., McCarthy, K., \& Rodriguez, S. (2006). Everyday magical powers: The role of apparent mental causation in the overestimation of personal influence. Journal of Personality and Social Psychology, 91, 218-231.

Rachman, S. (1997). A cognitive theory of obsessions. Behaviour Research and Therapy, 35, 793-802. 
Radloff, L. S. (1977). The CES-D Scale: A self-report depression scale for research in the general population. Applied Psychological Measurement, 1, 385-401. Reuven-Magril, O., Dar, R., \& Liberman, N. (2008). Illusion of control and behavioral control attempts in Obsessive-Compulsive Disorder. Journal of Abnormal Psychology, 117, 334-341.

Ridderinkhof, K. R., van den Wildenberg, W. P. M., Segalowitz, S. J., \& Carter, C. S. (2004). Neurocognitive mechanisms of cognitive control: The role of prefrontal cortex in action selection, response inhibition, performance monitoring, and reward-based learning. Brain and Cognition, 56, 129-140.

Riddle, A. S., Blais, M. R., Hess, U. (2002). A multi-group investigation of the CES-D's measurement structure across adolescents, young adults and middle-aged adults, CIRANO, Montreal (QC) (2002) Working Paper No. 2002s-36.

Rufer, M., Fricke, S., Held, D., Cremer, J., \& Hand, I. (2006). Dissociation and symptom dimensions of obsessive-compulsive disorder: A replication study. European Archives of Psychiatry and Clinical Neuroscience, 256, 146-150.

Salkovskis, P. M. (1985). Obsessional-compulsive problems: A cognitive-behavioural analysis. Behaviour Research and Therapy, $23,571-583$.

Summerfeldt, L. J., Huta, V., \& Swinson, R. P. (1998). Personality and obsessive-compulsive disorder. In R. P. Swinson, M. M. Antony, S. Rachman, \& M. A. Richter (Eds.), Obsessive-compulsive disorder: Theory, research, and treatment (pp. 79-119). New York: Guilford Press.

Synofzik, M., Vosgerau, G., \& Newen, A. (2008). Beyond the comparator model: A multifactorial two-step account of agency. Consciousness and Cognition, 17, 219-239.

Szechtman, H., \& Woody, E. Z. (2004). Obsessive-compulsive disorder as a disturbance of security motivation. Psychological Review, 111, 111-127.

Tolin, D. F., Brady, R. E., \& Hannan, S. (2008). Obsessional beliefs and symptoms of obsessive-compulsive disorder in a clinical sample. Journal of Psychopathology and Behavioral Assessment, 30, 31-42.

Tolin, D. F., Woods, C. M., \& Abramowitz, J. S. (2003). Relationship between obsessive beliefs and obsessive-compulsive symptoms. Cognitive Therapy and Research, 27, 657-669.

Ursu, S., Stenger, V. A., Shear, M. K., Jones, M. R., \& Carter, C. S. (2003). Overactive action monitoring in obsessive-compulsive disorder. Psychological Science, $14,347-353$

Vallacher, R. R., \& Wegner, D. M. (1985). A theory of action identification. Hillsdale, NJ: Lawrence Erlbaum.

Vallacher, R. R., \& Wegner, D. M. (1989). Levels of personal agency: Individual variation in action identification. Journal of Personality and Social Psychology, 57, 660-671

van der Weiden, A., Aarts, H., \& Ruys, K. (2010). Reflecting on the action or its outcome: Behavior representation level modulates high level outcome priming effects on self-agency experiences. Consciousness and Cognition.

Wegner, D. M. (2002). The illusion of conscious will. Cambridge, MA: MIT Press.

Wegner, D. M., \& Sparrow, B. (2004). Authorship processing. In M. Gazzaniga (Ed.), The new cognitive neurosciences (3rd ed., pp. 1201-1209). Cambridge, MA: MIT Press.

Wegner, D. M., \& Wheatley, T. P. (1999). Apparent mental causation: Sources of the experience of will. American Psychologist, 54, $480-492$.

Woody, E. Z., Lewis, V., Snider, L., Grant, H., Kamath, M., \& Szechtman, H. (2005). Induction of compulsive-like washing by blocking the feeling of knowing: An experimental test of the security-motivation hypothesis of obsessive-compulsive disorder. Behavioral and Brain Functions, 1, 1-11.

Woody, E., \& Szechtman, H. (2000). Hypnotic hallucinations and yedasentience. Contemporary Hypnosis, 17, 26-31.

Zermatten, A., \& Van der Linden, M. (2008a). Impulsivity in non-clinical persons with obsessive-compulsive symptoms. Personality and Individual Differences, 44, 1824-1830.

Zermatten, A., \& Van der Linden, M. (2008b). Phenomenal characteristics of memories of daily actions in checking-prone individuals. Applied Cognitive Psychology, 22, 1099-1112.

Zermatten, A., Van der Linden, M., Jermann, F., \& Ceschi, G. (2006). Validation of a French version of the Obsessive-Compulsive Inventory - Revised in a nonclinical sample. European Review of Applied Psychology, 56, 151-208.

Zermatten, A., Van der Linden, M., Larøi, F., \& Ceschi, G. (2006). Reality monitoring and motor memory in checking-prone individuals. Journal of Anxiety Disorders, 20, 580-596. 